VIrGinia Pianessole Piassarolu'

ELLEN HARDY ${ }^{2 \dagger}$

Nilva FerReira de ANDRADE ${ }^{3}$

Néville de Olivelra FerReira ${ }^{4}$

Maria José DuARTE Osis ${ }^{5}$

Artigo original

Palavras-chave

Assoalho pélvico/fisiopatologia Modalidades de fisioterapia

Terapia por estimulação elétrica

Eletromiografia

Disfunção sexual fisiológica

Qualidade de vida

Questionários

Keywords

Pelvic floor/physiopathology

Physical therapy modalities

Electric stimulation therapy

Electromyography

Sexual dysfunction, physiological

Quality of life

Questionnaires

Correspondência:

Maria José Duarte Osis

Centro de Pesquisas em Saúde Reprodutiva de Campinas

(CEMICAMP)

Caixa Postal 618

(EPP 13084-971 - Campinas (SP), Brosil

E-mail:miosis@cemicamp.org.br

Recebido

$8 / 7 / 09$

Aceito com modificaçôes

$21 / 5 / 10$

\section{Treinamento dos músculos do assoalho pélvico nas disfunções sexuais femininas}

\author{
Pelvic floor muscle training in female sexual dysfunctions
}

\section{Resumo}

OBJETIVO: avaliar o efeito do treinamento dos músculos do assoalho pélvico (TMAP) sobre as disfunções sexuais femininas. MÉTODOS: para esse ensaio clínico com abordagem antes e depois, foram incluídas 26 mulheres que apresentavam diagnóstico de disfunção sexual (transtorno de desejo sexual, de excitação, orgástico e/ ou dispareunia). As participantes foram avaliadas antes, na metade (após cinco sessões) e ao final do tratamento (após dez sessões), por meio da palpação vaginal bidigital (avaliação da força dos músculos do assoalho pélvico-MAP), eletromiografia (EMG) intravaginal (captação das amplitudes de contração dos MAP) e Female Sexual Function Index (FSFI), questionário de avaliação da função sexual. As mulheres foram submetidas ao TMAP em diferentes posições, por dez sessões (uma ou duas vezes na semana). Para análise estatística, utilizou-se frequências absolutas e relativas para características clínicas e força dos MAP. Empregou-se teste de Friedman para comparação dos escores dos domínios do FSFI e valores da EMG, t de Student para associação entre esses valores e características das mulheres e Wilcoxon para modificação percentual da EMG. O teste Mann-Whitney permitiu comparar esses valores com características clínicas. Para correlacionar os valores da EMG com escore total médio, utilizou-se teste de correlação de Spearman. Adotou-se nível de significância de $p<0,05$. RESULTADOS: foi observada melhora significativa $(p<0,0001)$ dos escores do FSFI ao final do tratamento quando comparado às avaliações inicial e intermediária. Em relação à EMG, as amplitudes das contrações fásicas e tônicas aumentaram significativamente ( $p<0,0001)$ ao longo do tratamento. Houve aumento na força do assoalho pélvico, com 69\% das mulheres apresentando grau 4 ou 5 na avaliação final e melhora total das queixas sexuais. CONCLUSÕES: o TMAP resultou na melhora da força muscular e amplitudes de contração pela EMG, com melhora na função sexual, o que indica que essa abordagem terapêutica pode ser utilizada com sucesso no tratamento das disfunções sexuais femininas.

\section{Abstract}

PURPOSE: to evaluate the effect of pelvic floor muscle training (PFMT) on female sexual dysfunctions. METHODS: twentysix women with a diagnosis of sexual dysfunction (sexual desire, arousal, orgasmic disorders and/or dyspareunia) were included in a clinical trial with a before/after approach. The assessment was carried out before, during lafter five sessions) and at the end of the treatment (after ten sessions) by two-digit palpation (assessment of pelvic floor muscle, PFM, strength), intravaginal electromyography (EMG) (capture of PFM contraction amplitudes) and Female Sexual Function Index (FSFI, a questionnaire for the evaluation of sexual function). The women underwent PFMT in different positions for ten sessions (once or twice a week). For statistical analysis, absolute and relative frequencies were used for clinical characteristics and PFM strength. The Friedman test was used to compare the FSFI domain scores and EMG values, the Student's t-test was used to determine the association between these values and the characteristics of the women, and the Wilcoxon test for percent modification of the EMG. The Mann-Whitney test permitted us to compare these values with clinical characteristics. The Spearman correlation test was used to correlate the EMG values with mean total score. Results

\section{Serviço de Fisioterapia e Ambulatório de Sexologia do Centro de Atenção Integral à Saúde da Mulher (CAISM) da Universidade} Estadual de Campinas - UNICAMP - Campinas (SP), Brasil.

' Pós-graduanda (Mestrado) do Curso de Tocoginecologia da Faculdade de Ciências Médicas da Universidade Estadual de Campinas - UNICAMP - Campinas (SP), Brasil.

${ }^{2}$ In memoriam; Pesquisadora Sênior do Centro de Pesquisas em Saúde Reprodutiva de Campinas - CEMICAMP - Campinas (SP).

Brasil; Professora do Curso de Pós-graduação em Tocoginecologia da Faculdade de Ciências Médicas da Universidade Estadual de Campinas - UNICAMP - Campinas (SP), Brasil.

${ }^{3}$ Ginecologista Sexóloga do Departamento de Tocoginecologia do Centro de Atenção Integral à Saúde da Mulher (CAISM) da Universidade Estadual de Campinas - UNICAMP - Campinas (SP), Brasil.

${ }^{4}$ Fisioterapeuta Supervisora do Curso de Especialização em Fisioterapia Aplicada à Saúde da Mulher do Centro de Atenção Integral à Saúde da Mulher da Universidade Estadual de Campinas - UNICAMP - Campinas (SP), Brasil; Pós-Graduanda (Doutorado) do Curso de Tocoginecologia da Faculdade de Ciências Médicas da Universidade Estadual de Campinas - UNICAMP - Campinas (SP), Brasil.

${ }_{5}^{5}$ Pesquisadora Sênior do Centro de Pesquisas em Saúde Reprodutiva de Campinas - CEMICAMP - Campinas (SP), Brasil; Professora Colaboradora do Curso de Pós-graduação em Tocoginecologia da Faculdade de Ciências Médicas da Universidade Estadual de Campinas - UNICAMP - Campinas (SP), Brasil. 
were considered statistically significant if $p<0.05$. RESULTS: a significant improvement $(p<0.0001)$ of FSFI scores was observed at the end of treatment compared to the values observed before and in the middle of treatment. Regarding the EMG, the amplitudes of tonic and phasic contractions increased significantly during treatment ( $p<0.0001)$. Pelvic floor strength increased, which $69 \%$ of the women presenting grade 4 or 5 at the end of treatment, with a total improvement of sexual complaints. CONCLUSIONS: the PFMT improved muscle strength and electromyography contraction amplitudes, with improved sexual function, indicating that this physiotherapy approach may be successfully used for the treatment of female sexual dysfunctions.

\section{Introdução}

A disfunção sexual feminina é um fenômeno frequente. Resultados da Pesquisa Nacional de Saúde e Vida Social (NHSLS) mostram que entre 30 e $50 \%$ de mulheres americanas têm algum tipo de disfunção sexual ${ }^{1}$. No Brasil, o Estudo da Vida Sexual do Brasileiro (EVSB), no qual foram pesquisadas 3.148 mulheres em 18 cidades, observou que $51 \%$ delas referiam alguma disfunção sexual ${ }^{2}$. Em estudo semelhante, foi encontrado $49 \%$ das mulheres com pelo menos uma disfunção sexual, sendo o desejo sexual hipoativo $(26,7 \%)$ o mais encontrado seguido de dispareunia $(23,1 \%)$ e disfunção orgástica $(21 \%)^{3}$, mas a disfunção sexual feminina ainda é pouco estudada ${ }^{4}$.

As causas de disfunção sexual nas mulheres são multifatoriais, envolvendo aspectos físicos, psicológicos, sociais ou até mesmo sendo de causa desconhecida. As mais apontadas na literatura são a idade (acima de 44 anos), o déficit de estrogênio pela menopausa, as cirurgias vaginais, as disfunções sexuais do parceiro, a crença religiosa, o desemprego e uma baixa percepção da qualidade de vida ${ }^{5-7}$. Além disso, fadiga, consumo de álcool ou drogas, gravidez, doenças crônicas e o desuso da musculatura perineal também têm sido mencionados como causas de disfunção ${ }^{8}$.

O desuso, a debilidade e a hipotonicidade dos músculos do assoalho pélvico (MAP) contribuíam para a incapacidade orgástica, e o treinamento destes tinha efeito positivo na vida sexual de mulheres ${ }^{9}$. Com relação ao transtorno orgástico, o treinamento dos músculos do assoalho pélvico (TMAP) poderia promover melhora, pois, com o aumento da força dos músculos que se inserem no corpo cavernoso do clitóris ${ }^{10}$, haveria melhor resposta do reflexo sensório-motor (contração involuntária dos MAP durante o orgasmo $)^{11}$, auxiliando na excitação e no orgasmo. Além disso, a melhora do fluxo sanguíneo pélvico, da mobilidade pélvica e da sensibilidade clitoriana após TMAP, potencializaria não só a excitação, mas também a lubrificação vaginal e o orgasmo $o^{12-14}$.

Assim, ambos TMAP e conscientização dos MAP têm sido apontados como técnicas auxiliares no tratamento das disfunções sexuais femininas por alterarem de maneira positiva a vida sexual ${ }^{9,13,15-19}$. O tratamento com esse foco frequentemente promoveria o aumento do desejo sexual com maior possibilidade de melhorar a excitação ${ }^{15,20}$.
Apesar dessas hipóteses, poucos estudos utilizaram questionários objetivos para avaliação da função sexual feminina após a aplicação dessa técnica fisioterapêutica (TMAP) e os correlacionaram à função muscular dos MAP $^{16,18,19}$. Além disso, são escassos os estudos que tentaram verificar quais mudanças ocorreriam nas diversas disfunções sexuais. Este artigo teve por objetivo avaliar o efeito do treinamento da musculatura do assoalho pélvico sobre as disfunções sexuais femininas.

\section{Métodos}

Foi realizado um ensaio clínico com abordagem antes e depois, no qual cada mulher era controle de si mesma. Foram selecionadas 45 mulheres no período de Fevereiro de 2008 a Maio de 2009, após a aprovação do projeto pelo Comitê de Ética em Pesquisa da Universidade Estadual de Campinas (UNICAMP).

As mulheres selecionadas apresentavam diagnóstico de disfunção sexual (transtorno de desejo, excitação, orgástico e dispareunia), segundo critérios da Classificação Estatística Internacional de Doenças e Problemas Relacionados à Saúde $(\mathrm{CID}-10)^{21}$, sendo avaliadas por uma ginecologista/sexóloga no Ambulatório de Sexologia do Centro de Atenção Integral à Saúde da Mulher (CAISM) da Universidade Estadual de Campinas (UNICAMP). Além do diagnóstico de disfunção sexual, as mulheres deveriam ter tido, pelo menos, uma relação sexual no último mês, idade entre 18 e 40 anos e parceiros estáveis. Não poderiam participar do estudo aquelas na menopausa ou climatério, com falência ovariana prematura, grávidas, mulheres com mais do que três gestações e/ou três partos, história pregressa de abuso e/ou estupro, diagnóstico de vaginismo, presença de diabetes, dislipidemias, hipertensão e/ou tratamento com hipotensores, depressão não tratada, que utilizassem anticonvulsivantes e/ou antidepressivos, submetidas a cirurgias pélvicas ou vaginais prévias, infecção ou perda urinária atuais, prolapso de órgãos pélvicos grau III ou IV, disfunção sexual do parceiro e ausência de contração dos MAP na avaliação física. As mulheres também não poderiam realizar nenhum outro tratamento para disfunção sexual durante a realização do estudo.

As mulheres elegíveis foram convidadas a participar do estudo após explicação dos objetivos do mesmo e dos procedimentos a serem seguidos. Aquelas que concordaram 
em participar foram encaminhadas à Seção de Fisioterapia e assinaram um termo de consentimento livre e esclarecido antes de iniciarem as avaliações e o TMAP.

Após, foi preenchida uma ficha contendo perguntas sobre as características clínicas e sociodemográficas das mulheres, como idade, renda familiar, escolaridade, estado civil, religião, cor da pele, peso, altura, uso de anticoncepcional oral, número de gestações e trabalho fora de casa. Elas também responderam a um questionário de avaliação da função sexual antes, na metade e após o treinamento dos MAP. O questionário utilizado foi o Female Sexual Function Index (FSFI), já validado no Brasil ${ }^{22}$. Ele é autorrespondido e composto por 19 questões, que contemplam seis domínios da resposta sexual: desejo, excitação, lubrificação, orgasmo, satisfação e dor/desconforto. A pontuação de cada questão é individual, variando de 0 a 5 . Para se obter a pontuação do domínio é necessária a soma de questões correspondentes a cada domínio, multiplicadas pelo fator de correção. Por meio da soma dos escores dos domínios, obtém-se o escore total, que apresenta valores mínimos de 2 e máximo de 36, sendo os maiores valores associados a uma melhor função sexual ${ }^{23}$.

Após preencherem o FSFI, as mulheres foram submetidas a um exame físico, na posição de decúbito dorsal (quadris e joelhos semifletidos), com intuito de avaliar a função dos MAP, por meio da graduação de força muscular e da eletromiografia (EMG). Para avaliação do grau de força, foi utilizado o toque vaginal (bi-digital), sendo considerados os graus de acordo com a escala de Oxford Modificada ${ }^{24}$, que variam de 0 a 5 Pelvic floor muscle assessment: the PERFECT scheme. Para captação da atividade mioelétrica, utilizou-se um eletrodo intravaginal conectado a um eletromiógrafo (Miotool 200, Miotec, Porto Alegre, RS), que converte a contração muscular em valores contínuos expressos em microvolts $(\mu \mathrm{V})$. O protocolo de avaliação eletromiográfica consistia em captar os valores do tônus muscular em repouso e dos valores médios e máximos das contrações fásicas e tônicas.

O protocolo de TMAP pélvico foi realizado na Seção de Fisioterapia do CAISM/UNICAMP e consistia em realizar os exercícios em grupo, por 10 sessões, 1 ou 2 vezes por semana, durante 50 minutos. Os exercícios dos MAP foram realizados em diversas posições (totalizando dez): decúbito dorsal, lateral e ventral; na posição de quatro apoios; sentada na cadeira e na bola; e em pé de frente ao espelho. Foram solicitadas, para cada posição, cinco contrações fásicas (rápidas) e cinco contrações tônicas (sustentadas) por dez segundos, com um período de relaxamento de dez segundos entre cada contração, totalizando, ao final de cada sessão, cerca de cem contrações. Todas as mulheres recebiam uma cartilha com orientações domiciliares, contendo explicações dos exercícios praticados para serem realizados uma vez ao dia.
O grau de força, a atividade eletromiog ráfica e o questionário de função sexual foram repetidos após a quinta sessão (avaliação intermediária) e ao final do tratamento (avaliação final), e todas as variáveis avaliadas sempre foram realizadas pelo mesmo investigador. Desse modo, ao encerrar as dez sessões, as participantes retornaram ao Ambulatório de Sexologia para nova avaliação da ginecologista/sexóloga. As mulheres que não apresentaram mais nenhuma queixa sexual receberam alta e às que ainda permaneceram com alguma queixa foi oferecida terapia sexual como forma de complementação ao tratamento para disfunção sexual.

Para análise estatística das características clínicas e epidemiológicas foram utilizados: média, desvio padrão $( \pm \mathrm{DP})$ e frequências relativas e absolutas. $\mathrm{Na}$ comparação dos escores médios dos domínios do questionário de função sexual ao longo do tratamento (entre as três avaliações), foi utilizado o teste não-paramétrico de Friedman para medidas repetidas, seguido do teste de Bonferroni para comparações múltiplas. Para avaliar a associação entre esses valores com as características das mulheres foi utilizado o teste $t$ de Student.

Foi utilizado o teste ANOVA de Friedman para medidas repetidas, seguido do teste de Bonferroni para comparações múltiplas, quando avaliados os valores medianos da atividade eletromiográfica ao longo do tratamento. Também se calculou a modificação percentual dos valores médios da atividade eletromiográfica entre três avaliações realizadas, utilizando o teste não-paramétrico de Wilcoxon pareado. Esses valores também foram comparados às características das mulheres por meio do teste não-paramétrico de Mann-Whitney.

Para análise da força do assoalho pélvico ao longo do tratamento, utilizou-se somente a expressão das frequências relativas e absolutas, e para correlacionar os valores médios da atividade eletromiográfica com o escore total médio, utilizou-se o teste de correlação de Spearman. O nível de significância estatística adotado foi de $5 \%(\mathrm{p}<0,05)$ e a análise dos dados foi realizada por meio do software SAS (versão 9.1.3, SAS Institute Inc., Cary, USA).

\section{Resultados}

Das 45 pacientes selecionadas e convidadas a participar do estudo, 14 foram excluídas por não comparecerem à avaliação inicial e 5 desistiram durante o estudo. Por esse motivo, apenas 26 foram introduzidas no estudo.

A média de idade das 26 participantes foi de 30,5 anos $( \pm 5,4 \mathrm{DP})$; a renda familiar média foi de $\mathrm{R} \$ 978,60$ $( \pm 451,2 \mathrm{DP})$ e a média do índice de massa corpórea (IMC) foi de $25,7 \mathrm{~kg} / \mathrm{m}^{2}( \pm 4,9 \mathrm{DP})$. A grande maioria (96\%) era casada ou vivia em união e $58 \%$ relatou ter completado o ensino médio. A maior parte declarou cor da pele não-branca 
(65\%), referiu ser católica (61\%), tinha tido, no máximo, duas gestações (81\%), não utilizava anticoncepcional oral $(70 \%)$ e, na avaliação física, somente $12 \%$ apresentava prolapso grau I ou II. Pouco mais da metade $(58 \%)$ das mulheres referiu não trabalhar fora de casa.

Em relação aos diagnósticos de disfunção sexual encontrados na avaliação inicial, o transtorno orgástico $(35 \%)$ e o transtorno de desejo (35\%) foram os mais comuns. Os outros diagnósticos apresentaram mais de uma queixa sexual associada. Das 26 mulheres que realizaram o TMAP, 69\% receberam alta do Ambulatório de Sexologia por melhora total das queixas sexuais. $\mathrm{O}$

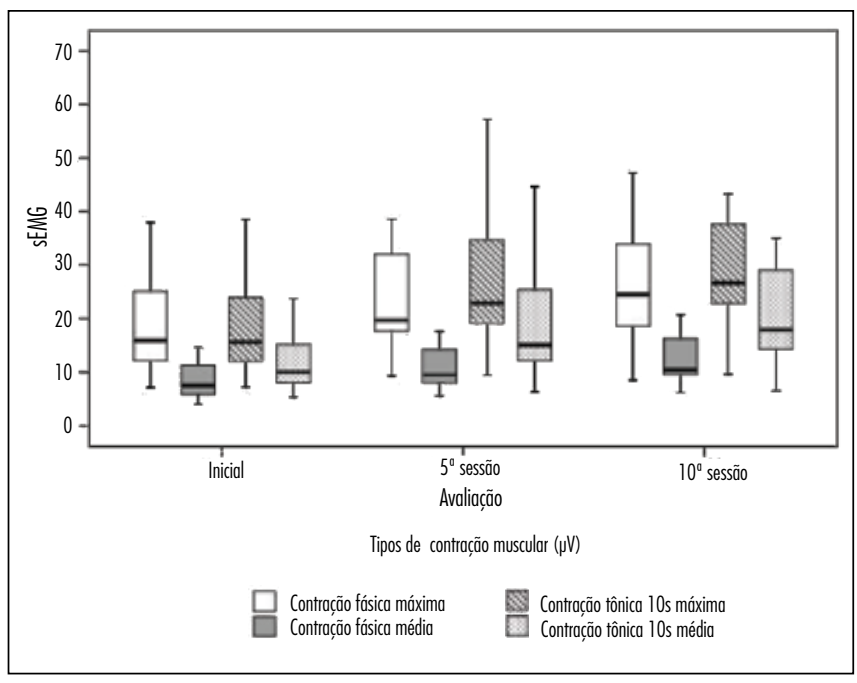

Figura 1 - Comparação entre as medianas das amplitudes das contrações da EMG ao longo do tempo restante $(31 \%)$ foi encaminhado para terapia sexual em grupo, realizada no ambulatório pela ginecologista/sexóloga. Das mulheres que foram encaminhadas à terapia, a metade delas apresentava transtorno de desejo, somente uma com transtorno orgástico e o restante apresentou a associação de mais de uma queixa.

$\mathrm{Na}$ Tabela 1 são apresentadas as médias ( $\pm \mathrm{DP})$ dos escores de cada domínio do questionário FSFI comparando resultados dos três momentos avaliados, observando-se melhora significativa em todos os domínios entre uma avaliação e outra. A média do escore total variou de 18,5 na avaliação inicial para 25,6 na avaliação intermediária e 30,3 na avaliação final. Não houve associação entre as variações do escore total em relação às características das mulheres.

Foram detectadas diferenças significativas ao comparar os valores eletromiográficos medianos das contrações fásicas e tônicas (média e máxima) encontrados nas avaliações inicial, intermediária e final, conforme demonstrado na Figura 1. Também foi observada diferença em relação à variação percentual dos valores da EMG na comparação entre as avaliações inicial, intermediária e final (Tabela 2). O tônus em repouso se manteve com o mesmo valor mediano de 3,9 $\mu \mathrm{V}$ durante todas as avaliações.

A evolução do grau de força dos MAP é visualizada na Tabela 3. Na avaliação inicial, $85 \%$ das mulheres apresentaram força de grau 1 ou 2 . Já na avaliação intermediária, $77 \%$ tiveram grau 3 ou 4 e, ao final do tratamento, quase todas as mulheres (69\%) apresentaram força dos MAP grau 4 ou 5 , demonstrando uma melhora de pelo menos 2 graus.

Tabela 1 - Média dos escores do FSFI ao longo do tempo

\begin{tabular}{|c|c|c|c|c|}
\hline \multirow{3}{*}{ Domínios do FSFI } & \multicolumn{3}{|c|}{ Avaliações } & \multirow{3}{*}{ Valor de $\mathrm{p}^{*}$} \\
\hline & Inicial & $5^{\mathrm{a}}$ sessão & $10^{\circ}$ sessc̃o & \\
\hline & Média ( \pm DP) & Média ( \pm DP) & Média ( \pm DP) & \\
\hline Desejo & $2,3(1,1)$ & $4(1)$ & $5(0,8)$ & $<0,0001$ \\
\hline Lubrificação & $2,7(0,9)$ & $4(0,7)$ & $4,9(0,7)$ & $<0,0001$ \\
\hline Excitação & $3,6(1,2)$ & $4,5(1)$ & $5,1(0,7)$ & $<0,0001$ \\
\hline Orgasmo & $2,8(1,3)$ & $3,9(1,3)$ & $4,7(1,1)$ & $<0,0001$ \\
\hline Total $(n=26)$ & $18,5(5,2)$ & $25,6(4,8)$ & $30,4(3,6)$ & $<0,0001$ \\
\hline
\end{tabular}

* Teste não-paramétrico de Friedman para medidas repetidas, seguido do teste de Bonferroni para comparações múltiplas.

Tabela 2 - Variação percentual média das amplitudes das contrações eletromiográficas ao longo do tempo

\begin{tabular}{|c|c|c|c|c|c|c|}
\hline \multirow{3}{*}{ Amplitudes das contrações } & \multicolumn{6}{|c|}{ Avaliaçãó } \\
\hline & \multicolumn{2}{|c|}{ Inicial-média } & \multicolumn{2}{|c|}{ Média-final } & \multicolumn{2}{|c|}{ Inicial-final } \\
\hline & Média ( \pm DP) & Valor de $\mathrm{p}^{*}$ & Média ( \pm DP) & Valor de $\mathrm{p}^{*}$ & Média ( \pm DP) & Valor de $\mathrm{p}^{*}$ \\
\hline Contração fásica (média) & $42,6( \pm 42,3)$ & $<0,0001$ & $13,8( \pm 21,1)$ & $<0,0003$ & $59,7( \pm 47,6)$ & $<0,0001$ \\
\hline Contração fásica (máxima) & $42,5( \pm 51,4)$ & $<0,0001$ & $13,5( \pm 17,8)$ & $<0,0001$ & $59,2( \pm 54,9)$ & $<0,0001$ \\
\hline Contração tônica (10s, média) & $50,4( \pm 46,7)$ & $<0,0001$ & $17,5( \pm 13,3)$ & $<0,0001$ & $77,4( \pm 59,2)$ & $<0,0001$ \\
\hline Contração tônica (10s, máxima) & $46,9( \pm 45,4)$ & $<0,0001$ & $15,1( \pm 14,1)$ & $<0,0001$ & $67,7( \pm 49,5)$ & $<0,0001$ \\
\hline
\end{tabular}

* Teste não-paramétrico de Wilcoxon pareado. 
Não houve correlação significativa entre os valores da EMG e o escore total do FSFI avaliados nos três momentos do estudo.

\section{Discussão}

Os resultados indicam que as mulheres submetidas ao TMAP apresentaram melhora significativa nos escores da função sexual do questionário FSFI (em todos os domínios e no escore total) e nas amplitudes da EMG ao longo do tratamento. Além disso, todas tiveram aumento da força dos MAP e houve melhora das queixas sexuais da maioria delas.

Neste estudo, os métodos utilizados para avaliação dos MAP foram a palpação vaginal e a EMG. Apesar de a palpação vaginal ser um método subjetivo para avaliar a contração dos MAP e muitos estudos apresentarem resultados controversos questionando sua eficácia ${ }^{17}$, é um método simples, de baixo custo e pode ser reproduzido por qualquer examinador ${ }^{25}$. Alguns autores ${ }^{16,26}$ conseguiram verificar uma melhora da contração dos MAP utilizando a palpação vaginal antes e após o treinamento desses músculos. Já a EMG é uma das formas mais precisas e fidedignas para se obter uma resposta fisiológica específica dos potenciais de ação das fibras musculares e para se observar melhoras significativas após um determinado tratamento ${ }^{19,27}$. Observou-se uma melhora estatisticamente significativa das contrações dos MAP ao longo do estudo utilizando a EMG.

A avaliação da função sexual é muito complexa, pois a sexualidade é um fenômeno multidimensional, associado a fatores biológicos, psicológicos, socioculturais e por determinantes interpessoais. Segundo alguns autores ${ }^{22,28}$, o FSFI é um instrumento adequado para estudos epidemiológicos e clínicos, de fácil aplicação e que permite avaliar de forma objetiva a sexualidade feminina. Esses aspectos também foram notados neste estudo, com melhora significativa da função sexual em todos os domínios e no escore total do FSFI. Esses resultados são semelhantes aos observados em outro estudo que utilizou o questionário FSFI para avaliar o efeito da eletroestimulação e dos exercícios em mulheres com dispareunia e vaginismo ${ }^{19}$. Apesar de ambos os estudos demonstrarem melhora dos escores de todos os domínios do FSFI, não existem muitos estudos que avaliaram o efeito do treinamento dos MAP em mulheres que somente apresentam disfunção sexual, principalmente transtornos de desejo e excitação ${ }^{17,19,20,29}$.

Neste estudo não foi possível observar correlação significativa entre os escores do FSFI e os valores da EMG. Esse fato pode ter ocorrido devido ao número reduzido de mulheres estudadas. No entanto, a função muscular dos MAP das mulheres estudadas melhorou e tal resultado confirma as propostas de $\mathrm{Kegel}^{9}$, que já acreditava, em
Tabela 3 - Distribuição percentual das mulheres segundo a força dos músculos do assoalho pélvico ao longo do tempo

\begin{tabular}{lccc}
\hline \multirow{3}{*}{ Grau de força } & \multicolumn{3}{c}{ Avaliaç̃óes } \\
\cline { 2 - 4 } & Inicial & Intermediária & Final \\
\cline { 2 - 4 } & $\mathrm{n}(\%)$ & $\mathrm{n}(\%)$ & $\mathrm{n}(\%)$ \\
\hline 1 & $8(31)$ & - & - \\
2 & $14(54)$ & $5(19)$ & - \\
3 & $3(11)$ & $15(58)$ & $8(31)$ \\
4 & $1(4)$ & $5(19)$ & $12(46)$ \\
5 & - & $1(4)$ & $6(23)$ \\
Total & $26(100)$ & $26(100)$ & $26(100)$ \\
\hline
\end{tabular}

1952, que o desuso, a debilidade e a hipotonicidade dos MAP contribuíam para a incapacidade orgástica e que a reabilitação e o fortalecimento desses tinham efeito positivo na vida sexual de mulheres. Recente revisão da literatura ${ }^{13}$ e alguns estudos ${ }^{12,19}$ relatam que o tratamento fisioterapêutico melhora e potencializa o orgasmo. Bø et al. ${ }^{16}$ observaram uma redução significativa do número de mulheres que apresentavam problemas com a vida sexual e social, após seis meses de TMAP. Em estudo semelhante, Beji et al. ${ }^{18}$ também verificaram melhora do desejo sexual, do orgasmo e do desempenho durante a relação sexual entre mulheres que realizaram o TMAP.

A forma como o TMAP se associa à função orgástica e até mesmo à função sexual em geral ainda é especulativa. Acredita-se que o fortalecimento dos MAP, principalmente dos isquiocavernosos e bulboesponjosos, auxilia na excitação e na facilidade de atingir o orgasmo por terem suas inserções no corpo cavernoso do clitóris ${ }^{10}$. O que também justificaria a melhora significativa da queixa sexual após o fortalecimento dos MAP é o fato de a resposta orgástica da mulher ser um reflexo sensório-motor que leva a contrações dos músculos perineais durante o orgasmo ${ }^{11}$.

Neste estudo, foram observadas evidências de que outras disfunções sexuais, além da dispareunia e do vaginismo, como os transtornos de desejo, excitação e a satisfação sexual, podem ser beneficiadas com a realização de treinamento dos MAP. Esse fato pode ter ocorrido, pois os exercícios do assoalho pélvico também produzem um aumento na vascularização pélvica ${ }^{12,14}$ e na sensibilidade clitoriana, o que promoveria uma melhor excitação e lubrificação. Sabe-se que, apesar de não haver na literatura informações sobre modificações químicas e moleculares promovidas por TMAP especificamente, encontra-se que qualquer treinamento muscular é capaz de promover melhora da função muscular com grandes modificações vasculares. Ainda assim, a literatura revela que são incertos quais realmente são os fatores de crescimento angiogênicos e suas sinalizações que devem estar envolvidas no remodelamento vascular e na melhora da função muscular ${ }^{30}$. Alguns autores ${ }^{12,14}$ e recente revisão da literatura ${ }^{13}$ também relatam esse aumento do fluxo 
sanguíneo e da mobilidade pélvica, o que potencializa a excitação genital e orgástica. Além disso, a integração de aspectos biopsicossociais na função sexual e da associação de uma ou mais disfunções poderia explicar o fato da melhora do transtorno de desejo nas mulheres. Kaplan ${ }^{15}$ já dizia que existe uma forte integração entre fatores orgânicos e psíquicos e que, em geral, muitas mulheres não têm conhecimento da potencialidade no prazer e da localização do clitóris. Acredita-se também que não só o fortalecimento, mas também a conscientização e a propriocepção dessa musculatura promoveriam uma maior percepção da região perineal, melhorando assim a autoimagem da mulher, sua receptividade em relação à atividade sexual e a satisfação com seu desempenho.

Após o término do tratamento, a maioria das mulheres que participou deste estudo (69\%) recebeu alta do Ambulatório de Sexologia, sem necessitar de terapia sexual como tratamento complementar. Isso sugere o potencial da abordagem fisioterapêutica como coadjuvante no tratamento das disfunções sexuais em geral. No entanto, sabe-se que a terapia cognitivo-comportamental é uma das formas de tratamento mais utilizadas, pois identifica problemas causadores da disfunção e auxilia na ansiedade e nos mitos relacionados às questões sexuais ${ }^{31}$. Dessa forma, é possível pensar que a associação das duas técnicas poderia contribuir para se obter resultados ainda melhores no tratamento das mulheres com disfunção sexual.
O tamanho da amostra pode ser considerado uma limitação do estudo, contudo, esse número foi adequado para o cálculo estatístico empregado. O acréscimo de um grupo controle poderia promover melhores afirmações em relação ao efeito do TMAP nas disfunções sexuais, porém não foi o objetivo inicial do estudo. Diferentemente de outros estudos com períodos mais prolongados ${ }^{16,18}$, foi realizado um número menor de sessões (somente dez), num período médio de três meses. Mesmo assim, foi observada uma redução significativa de queixas, melhora da função sexual e dos valores de contração e da EMG dos MAP até mesmo na comparação da avaliação inicial com a quinta sessão. Apesar disso, o ideal é que houvesse um maior tempo de acompanhamento das participantes, o que deverá ser feito em um próximo estudo.

De modo geral, os resultados apresentados encorajam novas pesquisas a respeito do TMAP como parte do tratamento de diversos transtornos sexuais em mulheres. No entanto, a realização de novas pesquisas a respeito do TMAP no tratamento das disfunções sexuais, com um grupo controle e maior tempo de acompanhamento das mulheres deve ser encorajada para promover resultados mais expressivos com esse tipo de técnica. Conclui-se que, ao lado das abordagens já utilizadas, o tratamento fisioterapêutico pode ser um recurso valoroso para auxiliar na resolução das disfunções sexuais e na melhora na qualidade de vida dessas mulheres.

\section{Referências}

1. Laumann EO, Paik A, Rosen RC. Sexual dysfunction in the United States: prevalence and predictors. JAMA. 1999;281(6): 537-44.

2. Abdo $\mathrm{CH}$. Estudo da vida sexual do brasileiro. São Paulo: Bregantini; 2004.

3. Abdo $\mathrm{CH}$, Oliveira WM Jr, Moreira ED Jr, Fittipaldi JA. Prevalence of sexual dysfunctions and correlated conditions in a sample of Brazilian women - results of the Brazilian Study On Sexual Behavior (BSSB). Int J Impot Res. 2004; 16(2): 160-6.

4. Moreira Junior ED, Glasser D, Santos DB, Gingell C. Prevalence of sexual problems and related help-seeking behaviors among mature adults in Brazil: data from the Global Study of Sexual Attitudes and Behaviors. Sao Paulo Med J. 2005;123(5):234-41.

5. Weber AM, Walters MD, Schover LR, Mitchinson A. Vaginal anatomy and sexual function. Obstet Gynecol. 1995;86(6):946-9.

6. Lemack GE, Zimmern PE. Sexual function after vaginal surgery for stress incontinence: results of a mailed questionnaire. Urology. 2000;56(2):233-7.

7. Artiles Pérez V, Gutiérrez Sigler MD, Sanfélix Genovés J. Female sexual function and related factors. Aten Primaria. 2006;38(6): $339-44$

8. Masters WH, Johnson VE. A conduta sexual humana [Costa D, tradutor]. 3a ed. Rio de Janeiro: Civilização Brasileira; 1979.
9. Kegel A. Sexual functions of the pubococcygeus muscle. West J Surg Obstet Gynecol. 1952;60(10):521-4.

10. Chambless DL, Sultan FE, Stern TE, O'Neill C, Garrison S, Jackson A. Effect of pubococcygeal exercise on coital orgasm in women. J Consult Clin Psychol. 1984;52(1):1 14-8.

11. Shafik $A$. The role of the levator ani muscle in evacuation, sexual performance, and pelvic floor disorders. Int Urogynecol J Pelvic Floor Dysfunct. 2000; 11 (6):361-76.

12. Graber B, Kline-Graber $G$. Female orgasm: role of pubococcygeus muscle. J Clin Psychiatry. 1979;40(8):348-51.

13. Rosenbaum TY. Pelvic floor involvement in male and female sexual dysfunction and the role of pelvic rehabilitation in treatment: a literature review. J Sex Med. 2007;4(1):4-13.

14. Ma Y, Qin H. Pelvic floor muscle exercises may improve female sexual function. Med Hypotheses. 2009;72(2):223.

15. Kaplan HS. A nova terapia do sexo. 4a ed. Rio de Janeiro: Nova Fronteira; 1974.

16. Bø K, Talseth T, Vinsnes A. Randomized controlled trial on the effect of pelvic floor muscle training on quality of life and sexual problems in genuine stress incontinence women. Acta Obstet Gynecol Scand. 2000;79(7):598-603.

17. Bø K, Finckenhagen HB. Vaginal palpation of pelvic floor muscle strength: inter-test reproducibility and comparison between 
palpation and vaginal squeeze pressure. Acta Obstet Gynecol Scand. $2001 ; 80(10): 883-7$.

18. Beji NK, Yalcin O, Erkan HA. The effect of pelvic floor training on sexual function of treated patients. Int Urogynecol J Pelvic Floor Dysfunct. 2003;14(4):234-8.

19. Nappi RE, Ferdeghini F, Abbiati I, Vercesi C, Farina C, Polatti F. Electrical stimulation (ES) in the management of sexual pain disorders. J Sex Marital Ther. 2003;29 Suppl 1:103-10.

20. Etienne MA, Waitman MC. Disfunções sexuais femininas: a fisioterapia como recurso terapêutico. São Paulo: LPM; 2006.

21. Organização Mundial de Saúde. Classificação Estatística Internacional de Doenças e Problemas Relacionados à Saúde: CID-10 [Centro Colaborador da OMS para Classificação de Doenças em Português-CBCD, tradutor]. Genebra: Organização Mundial de Saúde; 2008.

22. Thiel RRC, Dambros M, Palma PCR, Thiel M, Riccetto CLZ, Ramos MF. Tradução para português, adaptação cultural e validação do Female Sexual Function Index. Rev Bras Ginecol Obstet. 2008;30(10):504-10.

23. Rosen R, Brown C, Heiman J, Leiblum S, Meston C, Shabsigh R, et al. The Female Sexual Function Index (FSFI): a multidimensional self-report instrument for the assessment of female sexual function. J Sex Marital Ther. 2000;26(2):191-208.

24. Laycok J, Jerwood D. Pelvic floor muscle assessment: the PERFECT scheme. Physiotherapy. 2001;87(12):631-42.
25. Romanzi L, Polaneczky M, Glazer HI. Simple test of pelvic muscle contraction during pelvic examination: correlation to surface electromyography. Neurourol Urodyn. 1999;18(6): 603-12.

26. Isherwood PJ, Rane A. Comparative assessment of pelvic floor strength using a perineometer and digital examination. BJOG. 2000;107(8):1007-11.

27. Rett MT, Simões JA, Herrmann V, Pinto CL, Marques AA, Morais SS. Management of stress urinary incontinence with surface electromyography-assisted biofeedback in women of reproductive age. Phys Ther. 2007;87(2): 136-42.

28. Blümel ME, Binfa EL, Cataldo AP, Carrasco VA, Izaguirre LH, Sarrá CS, et al. Índice de función sexual femenina: un test para evaluar la sexualidad de la mujer. Rev Chil Obstet Ginecol. 2004;69(2): $118-25$.

29. Dean N, Wilson D, Herbison P, Glazener C, Aung T, Macarthur C. Sexual function, delivery mode history, pelvic floor muscle exercises and incontinence: a cross-sectional study six years post-partum. Aus N Z J Obstet Gynaecol. 2008;48(3): 302-11.

30. Prior BM, Yang HT, Terjung RL. What makes vessels grow with exercise training? J Appl Physiol. 2004;97(3): 111 19-28.

31. Rosenbaum TY. Physiotherapy treatment of sexual pain disorders. J Sex Marital Ther. 2005;3 1 (4):329-40. 